\title{
An Advanced Strategy of Multi User Storage System for Cloud Computing
}

\author{
Pallam Reddy Venkata Subba Reddy, Sriharsha Vikruthi, E.V.N.Jyothi
}

\begin{abstract}
Cloud computing is an emerging model of business computing. In cloud computing, client can use and retrieve the services anytime by using any smart devices to manage complex computing processes and to access very large data storage. The developers have recognized the required of a multi USER storage system that can help in utilizing the cloud power by enhancing its functionality and improve its performance. In this paper, proposing the architecture of advanced Multi-user storage System which primarily focuses on the price negotiation mechanism between cloud users and providers. This system presents a perfect way for scalable and open systems that are changed dynamically. The model is based on cooperative and collaborative USERs and is managed. Also this architecture is designed to monitor the user's jobs while they are being processed.
\end{abstract}

Keywords: Fault detection module, Trust Decision Model, Virtual Cloud Environment

\section{INTRODUCTION}

Depend on the internet; the cloud computing strategy is determined. It uses a registering stage which will compose the advantages of the system. The cloud computing process will mainly depend on the storage servers. Hence the cloud storage will administrate at low cost [1]. This system will mainly used in the various applications and service forms. By using broadband network the cloud computing is implemented. This cloud computing will use the resources which is beneficial to the consumer or user. The cloud pursues a multi-occupant display in which figuring assets are pooled and the assets are progressively assigned according to the need of the customers [2]. This gives area freedom as the purchaser doesn't know about the correct physical area of an asset in the cloud. Security basic ongoing frameworks require working appropriately to evade disappointment, which can cause money related misfortune just as setbacks. So there is an expanded need to endure the flow for such sort of frameworks to be utilized with cloud foundation. For this reason exhibited a model for the adaptation to internal failure of ongoing applications running at cloud foundation. The fundamental system to accomplish the adaptation to internal failure is replication or repetition. This replication in the type of programming variations running on the various virtual machines.

Revised Manuscript Received on December 30, 2019.

* Correspondence Author

Pallamreddy Venkatasubbareddy, Associate Professor, Department Of Computer Science And Engineering, Pace Institute Of Technology And Sciences Ongole, Ap, India-523272

Sriharsha Vikruthi, Associate Professor, Department Of Computer Science And Engineering, Pace Institute Of Technology And Sciences Ongole, Ap, India-523272

E.V.N.Jyothi, Associate Professor, Department Of Computer Science And Engineering, Pace Institute Of Technology And Sciences Ongole, Ap, India-523272

(c) The Authors. Published by Blue Eyes Intelligence Engineering and Sciences Publication (BEIESP). This is an open access article under the CC BY-NC-ND license (http://creativecommons.org/licenses/by-nc-nd/4.0/)
Because of the replication, cost for leasing the cloud assets will increment. Be that as it may, it is truly required to keep away from the disastrous misfortune [3] Cloud computing is a model for enabling omnipresent, supportive, on-demand compose access to a typical pool of configurable figuring resources (e.g., frameworks, servers, storing, applications, and organizations) that can be immediately provisioned and released with unimportant organization effort or expert association affiliation. Distributed computing offers a practical answer for deal with the IT foundation in an adaptable and versatile way [4]. Distributed computing empowers programming applications, arrangement stages, even the processing assets to be made accessible on-request utilizing a compensation as-you-go model. This has drawn a ton of consideration towards the space as of late. Today a decent number of associations utilize the cloud for their everyday activities and the appropriation rate by others are additionally high [5]. Extra assets can be effectively provisioned or discharged according to the interest. In the cloud the asset accessibility show up basically boundless and assets can be asked for in any amount contingent on prerequisites. The cloud should deal with the scaling as required.

There are five attributes of cloud computing. The first is ondemand self-advantage, where a customer of organizations is given the required resources without human intervention and relationship with cloud provider. The subsequent trademark is extensive framework get to, which suggests resources can be gotten to from wherever through a standard part by slight or thick client stages such PDA, PC, and PC. Resource pooling is another trademark, which suggests the benefits are pooled with the true objective for multitenant to share the advantages. In the multi-inhabitant show, resources are distributed effectively to a customer and after the purchaser finishes it, it will in general be doled out to another respond to high resource demand. Not with standing whether the benefits are assigned to customers on intrigue, they don't have the foggiest thought regarding the zone of these doled out resources

\section{II.MODULES IN MULTI USER STORAGE SYSTEM}

Cloud computing enables the clients to store their information on the capacity area kept up by an outsider. When the information is transferred into the cloud the client loses its power over the information and the information can be altered by the assailants. The aggressor might be an outside. Unapproved get to is additionally a typical practice because of feeble access control. From figure (1) it can observe the block diagram of modules used in multi USER storage system. Multi USER storage system consists of the following modules: 


\section{An Advanced Strategy of Multi User Storage System for Cloud Computing}

- Data Owner Module

- $\quad$ File Upload Module with Encoded

- File Download Module with Decryption

- Rank Hunt Module

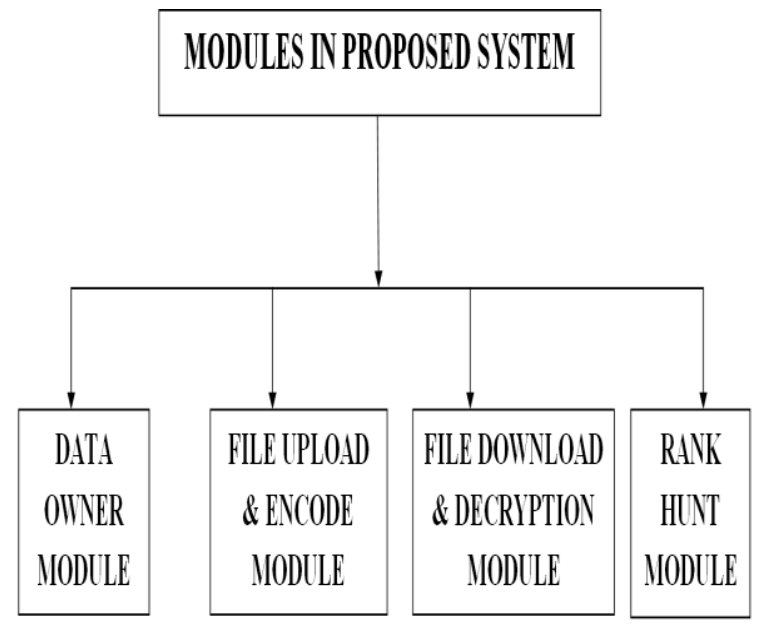

Fig. 1: MODULES IN MULTI USER STORAGE SYSTEM

\section{Data Owner Module}

In this module, the information proprietors ought to probably transfer the documents. The documents are encoded before the records are transferred to the cloud. The information proprietors are given an alternative to enter the watch words for the record that are transferred to the server. These watch words are utilized for the ordering reason which enables the chase to return esteems very rapidly. These documents when once accessible on the cloud, the information clients should be capable chase utilizing the catch phrases. The information proprietors will likewise be given a demand endorsement screen so they can favor or reject the demand that is gotten by the information clients.

\section{File Upload \& Encoded Module}

In this module, the information proprietors ought to most likely transfer the documents. The records are encoded before the documents are transferred to the cloud. The information proprietors are given an alternative to enter the catchphrases for the record that are transferred to the server. These catchphrases are utilized for the ordering reason which enables the chase to return esteems very rapidly. These documents when once accessible on the cloud, the information clients ought to most likely chase utilizing catchphrases. The information proprietors will likewise be given a demand endorsement screen so they can favor or reject the demands that are gotten by the information clients.

\section{File Download \& Decryption Module}

In this module the files are downloaded after decryption process. This system will provide security in effective way. The downloaded data will be saved in the server. The user has a chance to use the data that is saved in the cloud server.

\section{III. MULTIUSER STORAGE SYSTEM}

In cloud computing system, the system is monitored based on the user specification. Here an autonomic system is used by the user to develop the multi user technology. The inter relation between the multi user and the autonomic system will be very close to each other. The main intent of using the autonomic computing is to manage the system by itself. Based on the specifications, the system gives reliable and extendable outputs. Let us discuss bout the user platform in detail manner.

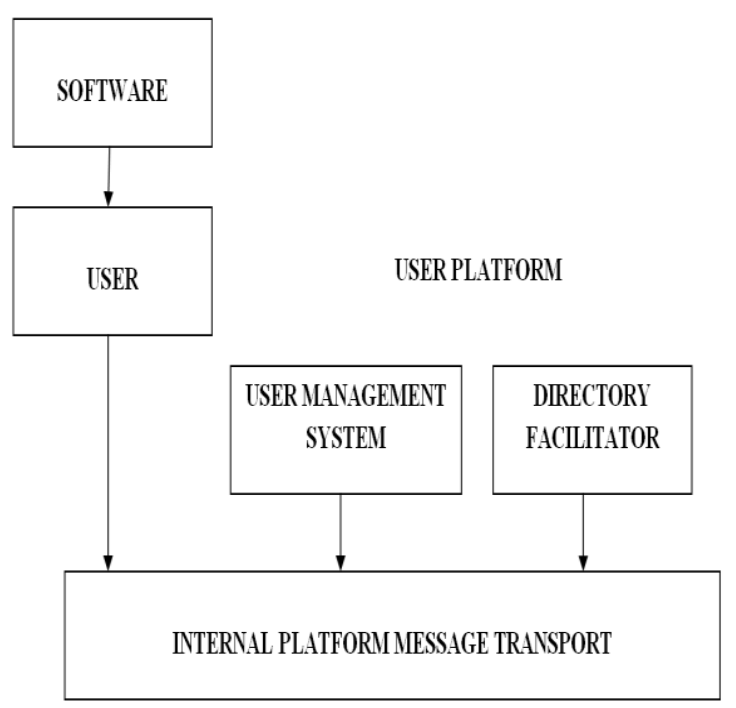

Fig. 2: AN USER PLATFORM

The above figure (2) shows the User platform architecture using FIPA model. The Foundation for Intelligent Physical Agents (FIPA) is worldwide associations that are sharing the endeavors to make attributes for basic operator advancements. The FIPA qualities forms rely upon two key explanations. The first is the expected time to get an assertion. The second explanation is just about the outside activities of framework parts which are referred to the execution data and inside plans ought to move engineers stage.

In the operational stage, complex tasks are separated into smaller undertakings and conveyed to various capable specialists at the lower levels. Cooperation among specialists in a MAS is for correspondence of acknowledge methods. The specialist order of our multi user storage system depends upon few supervisory spaces specialists like event open space, the gathering space, the group space and individual space. Each supervisory space operator speaks with four specialists: the supervisory user of the actors, the supervisory user of the activities, the supervisory user of the resources and finally the supervisory user of the tools. Every single one of these four specialists can administer different operators of lower chain of importance.

The cloud computing is distributed framework with an intricate accumulation of processing assets from various areas with various authoritative arrangements. This will increase the storage of cloud computing. In a multi-space condition, cloud has a large number of administration occurrences, finding cloud administrations are a big task owning to the way that there are countless administrations from various suppliers. This methodology utilized the utilization of self-sorting out operators for robotizing and organizing asset the board with promising profoundly powerful practices. The below figure (3) shows the architecture of multi user storage system. 


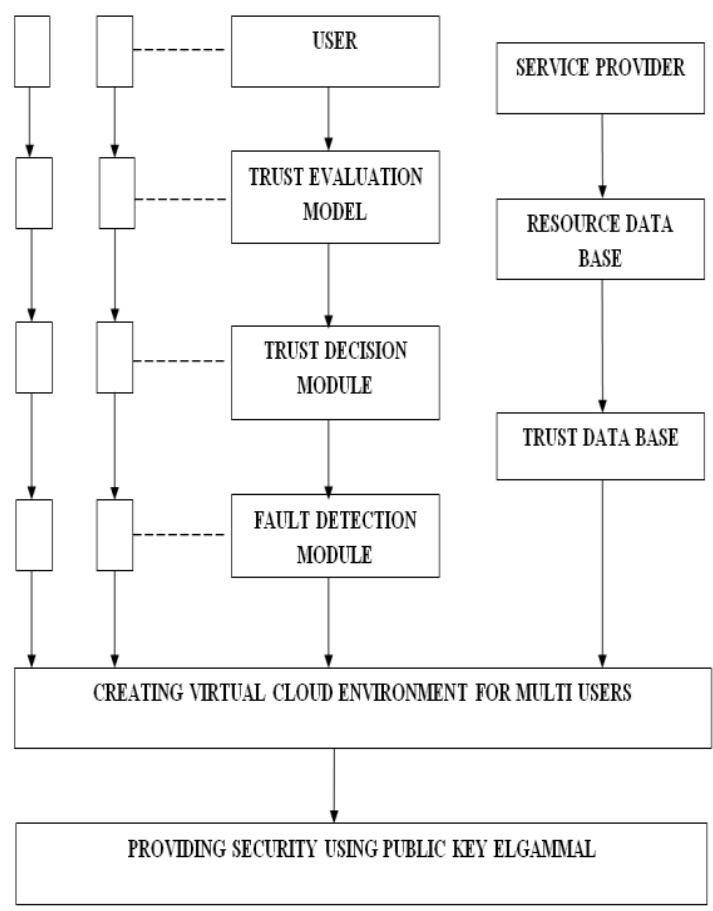

Fig. 3: MULTI USER STORAGE SYSTEM ARCHITECTURE

In this mainly user plays significant role. The trust evaluation model is used to evaluate the proposed model. Now the detection model will detect the system information which is obtained from the system. By using fault detection system the errors in the information will be detected. Now after fault detection a new environment is created by the cloud computing system. At last security is provided for the information by using public key elgamal.

The multi USER cloud computing is considered as the core of the cloud framework. It has the capacity to interface with operators that follow up for the benefit of the customers and suppliers. The intermediary is a specialist trough independent from anyone else; it facilitates the collaborations between the customers and suppliers operators, screens the running employments and stays up with the latest with the running procedure as shown in figure (3).

The intermediary goes about as an open or a private cloud administration. It fills in as center application or a PC between the cloud customers and the cloud suppliers. Typically the representative is considered as a database focus to the clients and the suppliers in the cloud condition. By and large it is a facilitator between at least two members to discover and choose the best assets to fit the customers employments. This is done when the cloud customers send their info documents which incorporate the sets of expectations to the USER. The specialist sends these documents to the best cloud suppliers, screens employments running, recovers the outcomes and sends it back to the customers.

In this paper, the multi USER cloud computing system has been moved from going about as a database putting away the suppliers details, taking care of the customers business to the suppliers and the occupations results from suppliers to the customers into a specialist chief that can control occasion creation; enrolling and evacuating every operator in the framework. The primary player charmed in cloud framework tasks is typically individuals who look in the cloud supplier's site and pick the USERs (on the off chance that these buys are appropriate). This issue is happened due to the short on a method for supporting arrangement interfaces by means of existing cloud suppliers to manage it powerfully. The proposed framework is a multi-operator framework that intends to communicate with the cloud condition in favor of cloud customers in order to get the records of all accessible cloud suppliers who can satisfy the client's determinations and to pick the best suppliers between them. Likewise, this framework plans to screen the occupations achievement and to give the operator trough (intermediary) with the correct criticisms about the activity procedure.

Cloud client portray their employments by decide the required equipment and programming particulars to achieve their occupations. The cloud supplier acquires the sets of expectations (demands) from customers, and contrasts the demand and the enlisted cloud suppliers and picks the most ideally equipped provider(s) for the activity. The dealer deals with the decisions that were obtained in second step and picks the best ones between them.

This kind of arranging is finished by the exchange time and cost. From that point forward, the representative contacts the operators that are in charge of the association between the dealer and the suppliers to ensure that the suppliers are accessible to get the new occupations. The specialist may have the benefits to part benefits between different specialist organizations to diminish the expense and accelerate the activity. The multi-USER storage system work builds up a structure that encourages the cooperation and coordination between the specialist organization and USER operators through the distributed computing condition.

\section{RESULTS}

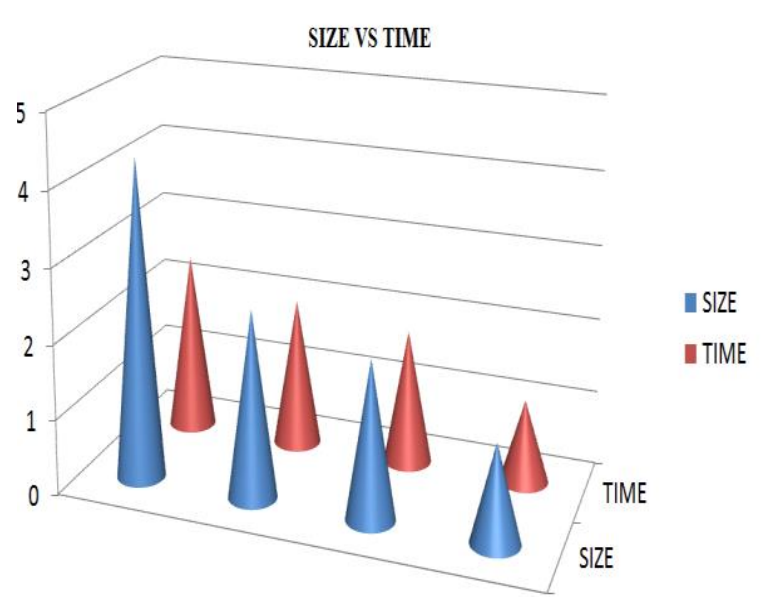

FIG. 4: SIZE AND TIME PERFORMANCE OF THIS MUTLI-CLOUD APPROACH

The above figure (4) shows the graph of advanced multi storage system. Here the proposed system occupies less area and less size.

Published By:

Blue Eyes Intelligence Engineering 


\section{An Advanced Strategy of Multi User Storage System for Cloud Computing}

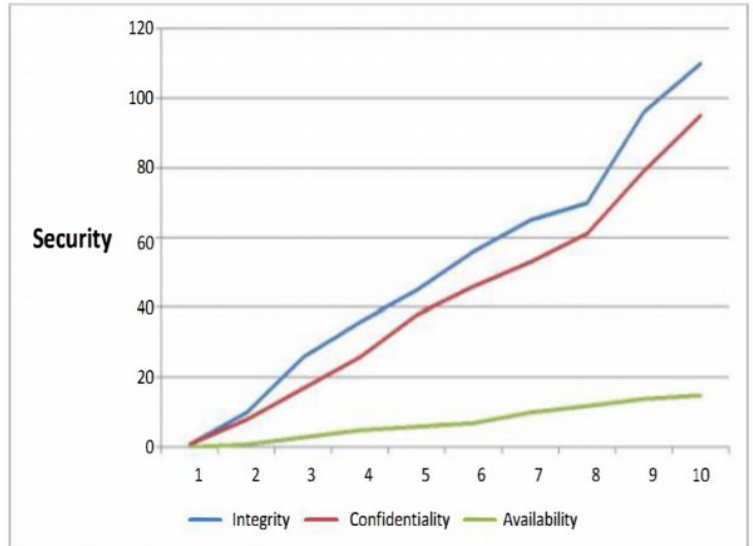

Fig. 5: SECURITY FACTOR OF THIS MUTLICLOUD APPROACH

The above figure (5) shows the graph of security factor. Here high level assessment is performed in the multi cloud approach and this is shown from below figure (6)

\begin{tabular}{|c|c|c|}
\hline \multirow[t]{3}{*}{ Security } & Integrity & $H$ \\
\hline & Confidentiality & H \\
\hline & Avallability & . \\
\hline \multirow[t]{3}{*}{ Feasibility } & Applicability & 0 \\
\hline & Businesss-Readiness & $\dagger$ \\
\hline & Ease of Use & 0 \\
\hline Regulation & Compliance & $\dagger$ \\
\hline
\end{tabular}

\section{Fig. 6: ASSESSMENT OF THIS MUTLI-CLOUD} APPROACH.

Basically, the consideration of this approach is based on the three considerations. The considerations are security, feasibility and regulation.

\section{CONCLUSION}

Cloud computing enables users to store their data in remote storage location. But data security is the major threat in cloud computing. The security provided to the system will be designed based on the storage. The both layers in the system will store the data in efficient way. The advanced multi user storage has many benefits which will detect the errors while processing the system. At last the proposed system gives confidentiality data and effective integration for the users.

\section{REFERENCES}

1. Chen Hong, Dianxi Shi, "Poster: A Control System Architecture with Cloud Platform for Multi-UAV Surveillance", 2018 IEEE SmartWorld, Ubiquitous Intelligence \& Computing, Advanced \& Trusted Computing, Scalable Computing \& Communications, Cloud \& Big Data Computing, Internet of People and Smart City Innovations

2. Hsin-Ya Chen , Pei-Yu Lee , Hsung-Pin Chang, "A Multi-tiered Storage Structure for Cloud Computing", 978-1-5090-3438-3/16 \$31.00 ( 2016 IEEE.
3. Khaled Riad, "Multi-Authority Trust Access Control For Cloud Storage", 978-1-5090-1256-5/16/\$31.00 (C2016 IEEE.

4. A. Manasa, S. Jessica Saritha, "An Evidence Multi-copy Dynamic Data Possession in Multi Cloud Computing System", 2016 international conference on communication and electronic systems.

5. Yuan Naiheng, Guo Yijun, Hao Jianjun , "Efficient Low-cost Storage Strategy in Multi-Cloud”, 2016 2nd IEEE International Conference on Computer and Communications.

6. Xiaolan Xie , Yang Li , "Trust Management Model of Cloud Computing Based on Multi-agent”, 2015 International Conference on Network and Information Systems for Computers.

7. ZHANG Yaling, JIA Zhipeng, WANG Shang ping, "A Multi-User Searchable Symmetric Encryption Scheme for Cloud Storage System", 2013 5th International Conference on Intelligent Networking and Collaborative Systems.

8. Mohammed, A. AlZain, Eric Pardede, Ben Soh\& James Thom, A 2012, 'Cloud Computing Security: From Single to Multi-clouds', hicss, 45th Hawaii International Conference on System Sciences, pp.54905499.

9. Amir Mohamed Talib , Rodziah Atan, Rusli Abdullah \& Masrah Azrifah Azmi Murad, "Security Framework of Cloud Data Storage Based on Multi Agent System Architecture - A Pilot Study", 978-14673-1090-1/12/\$31.00 C2012 IEEE

10. Yanjiang Yang, "Towards Multi-User Private Keyword Search for Cloud Computing”, 2011 IEEE 4th International Conference on Cloud Computing.

\section{AUTHOR'S PROFILES}

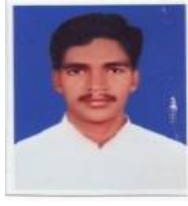

P.Venkata SubbaReddy, Working as Associate Professor in CSE Department of PACE Institute of Technology and Sciences, ONGOLE. He Has 17 Years of Teaching Experience. I Have published more than 20 papers in Various Journals and Expert in Paper Evaluation.

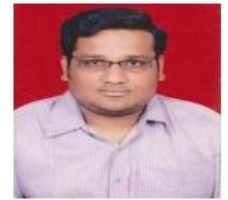

I Sriharsha Vikruthi, working as an Assistant Professor in CSE Dept. of Pace Institute of Technology \& Sciences. Currently pursuing Ph.D in CSE Department at Annamalai University. I have 10 years of experience in teaching for various Professional Institutions across India. So far I have published 12 papers in various Journals \& Conferences.

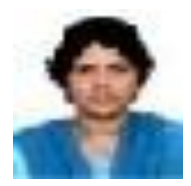

I E.V.N.Jyothi, working as an Assoc.Prof. in CSE Dept. of Pace Institute of Technology \& Sciences. I did M.Tech in CSE and currently doing Ph.D in CSE Department from Shri Jagdishprasad Jhabarmal Tibrewala University. I have 14 years of experience in teaching for various Professional Institutions across India. So far I have published 20 papers in various Journals \& Conferences. 